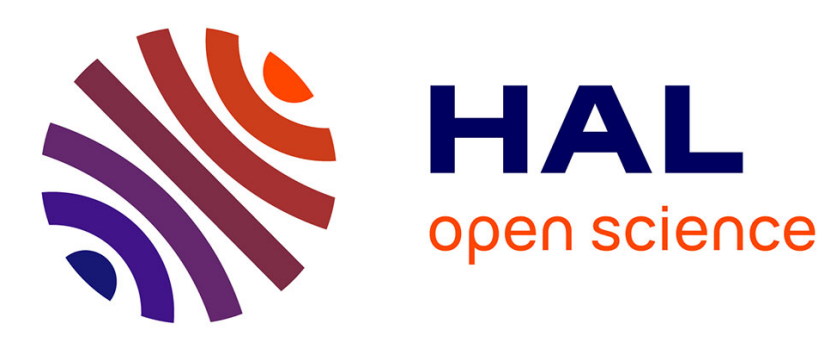

\title{
Excited states and energy transfer among DNA bases in double helices
}

D. Markovitsi, T. Gustavsson, F. Talbot

\section{To cite this version:}

D. Markovitsi, T. Gustavsson, F. Talbot. Excited states and energy transfer among DNA bases in double helices. Photochemical \& Photobiological Sciences , 2007, 6, pp.717-724. 10.1039/b705674e . hal-00162437

\section{HAL Id: hal-00162437 \\ https://hal.science/hal-00162437}

Submitted on 13 Jul 2007

HAL is a multi-disciplinary open access archive for the deposit and dissemination of scientific research documents, whether they are published or not. The documents may come from teaching and research institutions in France or abroad, or from public or private research centers.
L'archive ouverte pluridisciplinaire HAL, est destinée au dépôt et à la diffusion de documents scientifiques de niveau recherche, publiés ou non, émanant des établissements d'enseignement et de recherche français ou étrangers, des laboratoires publics ou privés. 


\section{Excited states and energy transfer among DNA bases in double helices}

Dimitra Markovitsi ${ }^{*}$, Thomas Gustavsson, Francis Talbot

Laboratoire Francis Perrin, CEA/DSM/DRECAM/SPAM - CNRS URA 2453,

CEA/Saclay, 91191 Gif-sur-Yvette, France, 


\section{Abstract:}

The study of excited states and energy transfer in DNA double helices has recently gained new interest connected to the development of computational techniques and that of femtosecond spectroscopy. The present article points out contentious questions regarding the nature of the excited states and the occurrence of energy transfer and shows how they are currently approached. Using as example the polymer poly(dA).poly(dT), composed of about 2000 adenine-thymine pairs, a model is proposed on the basis of time-resolved measurements (fluorescence decays, fluorescence anisotropy decays and fluorescence spectra, obtained with femtosecond resolution), associated to steady-state spectra. According to this qualitative model, excitation at $267 \mathrm{~nm}$ populates excited states that are delocalized over a few bases (excitons). Ultrafast internal conversion directs the excited state population to the lower part of the exciton band giving rise to fluorescence. Questions needing further investigations, both theoretical and experimental, are underlined with particular emphasis on delicate points related to the complexity and the plasticity of these systems. 


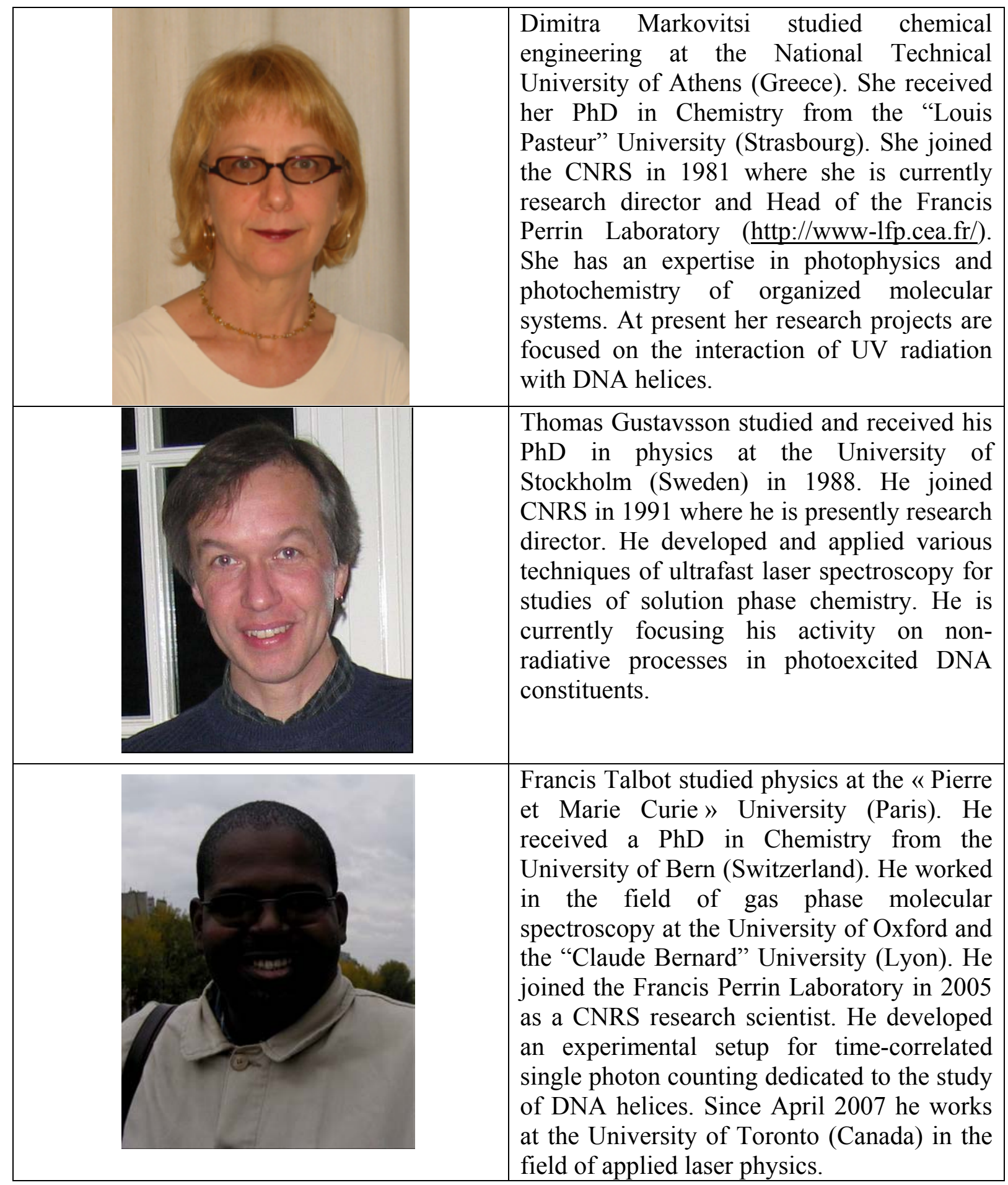




\section{Introduction}

It is presently well established that absorption of UV light by DNA triggers photochemical reactions which may induce carcinogenic mutations ultimately leading to skin cancer. This knowledge has given an important impulse to the field of DNA photochemistry. Thousands of publications deal with a large variety of aspects ranging from gas phase spectroscopy of nucleobases to the characterization of numerous photoproducts in vitro and in vivo. Despite such an intense research activity, the fate of the excitation energy within double helices is far from being clear. In connection to this problem, aspects such as the nature of the Franck-Condon (FC) states populated immediately upon photon absorption, the occurrence of excitation energy transfer, the nature of the fluorescent states or relaxation toward dark states are discussed. But very often controversial conclusions were reached, depending on the used methodology.

The aim of the present article is to point out contentious questions and show the way that these are approached currently (Section 1). In the following Sections, we examine the nature of the excited states as well as excitation energy transfer among bases using as example the polymer poly(dA).poly(dT), composed of about 2000 adenine-thymine pairs. The fluorescence decays and fluorescence anisotropy decays of this double strand, excited by femtosecond pulses and detected using fluorescence upconversion (FU) and time-correlated single photon counting (TCSPC), were recently published in a short communication. ${ }^{1}$ Here, we examine in detail these results. The study is enriched by the first time-resolved fluorescence spectra recorded for a DNA duplex in the sub-picosecond time scale. Moreover, we present global fits of the fluorescence signals recorded by FU and TCSPC, providing fluorescence decays over a large time-domain (100 fs - $5 \mathrm{~ns}$ ) deconvoluted for the instrumental response functions. In the discussion, the importance of considering both steadystate and time-resolved spectroscopic data is stressed and various subtle factors related to both experimental and conceptual difficulties are underlined. Finally, we comment on possible future developments.

\section{Old questions, recent approaches}

A main issue regarding the FC states of DNA is whether they are localized on single bases or delocalized over a certain number of them (excitons). This question was first addressed in the 1960s. Theoretical calculations, performed in the frame of the exciton theory, concluded that the excited states in DNA should be delocalized. ${ }^{2-4}$ At the same time, they 
predicted that the existence of exciton states should induce large spectral shifts with respect to non-interacting chromophores and generate a visible splitting of the UV absorption band located around $260 \mathrm{~nm} .{ }^{3}$ Since those features are not observed in the experimental absorption spectra of DNA, which instead closely resemble the sum of the spectra of the constituent bases, the hypothesis of localized excited states prevailed ${ }^{5}$ and guided subsequent studies dealing with the photophysics and the photochemistry of DNA.

In the meantime, the development of the computational techniques and a better knowledge of the excited states of monomeric bases, nucleosides and nucleotides allowed more precise calculations to be performed. The properties of FC excited states of model double helices were determined again in the frame of exciton theory, but now combining data from molecular dynamics simulations and quantum chemistry calculations and taking into account the spectral width of the chromophores. ${ }^{6-9}$ It was shown that, despite the structural fluctuations and the important dispersion of the excitation energy, dipolar coupling is capable of inducing delocalization of the FC excited states over a few bases. Moreover, the absorption spectra simulated under these conditions were found to be only slightly shifted with respect to the spectra of non-interacting chromophores and to exhibit no visible splitting. ${ }^{8,9}$ Thus, the argument against exciton states based on the discrepancy between calculated and experimental spectral shifts was proved to be incorrect.

From an experimental point of view, some recent investigations dealing with steadystate spectra of functionalized double helices discussed the delocalization of the FC states. One of them examined fluorescence excitation and emission spectra of duplexes containing 2aminopurines as a function of their relative position within double helices. ${ }^{10}$ Others were based on the changes observed in the spectra of steady-state UV absorption and circular dichroïsm recorded for a series of capped duplexes of various lengths. ${ }^{11-13}$ Both types of studies concluded on delocalization of the excitation over a few bases.

Transfer of excitation energy within double helices at room temperature has also been the object of several studies using mainly two different approaches. One of them consists in observing photosensitized fluorescence from an energy acceptor (intercalator or modified base). ${ }^{14-18}$ The problem is that such investigations provide information about excitation transfer between DNA bases and the energy acceptor, and the conclusions cannot necessarily be extrapolated to energy transfer occurring among natural bases. According to another approach, fluorescence of double helices containing only natural bases is observed. The related experiments are very delicate because the fluorescence quantum yield of DNA at room temperature is extremely low $\left(c a .10^{-4}\right) .{ }^{19}$ Thus, in 1977, Vigny and Ballini studied 
fluorescence from highly purified DNA but their result appeared to be puzzling due to the complexity of the system. ${ }^{20}$ Therefore, they underlined the necessity to work with helices composed of one type of base-pairs.

Fluorescence from model duplexes containing only natural bases with simple sequence, $(\mathrm{dA})_{\mathrm{n}} \cdot(\mathrm{dT})_{\mathrm{n}},(\mathrm{dAdT})_{\mathrm{n}} \cdot(\mathrm{dAdT})_{\mathrm{n}}$ or $(\mathrm{dCdG})_{\mathrm{n}} \cdot(\mathrm{dCdG})_{\mathrm{n}}$, was indeed studied later. ${ }^{21-24}$ In fact, Georghiou et al. examined the variation of the emission spectra and that of fluorescence anisotropy upon changing the excitation or the emission wavelength. They concluded that energy transfer among bases is not efficient and that emission stems from just either one type of base (thymine in $(\mathrm{dA})_{\mathrm{n}} \cdot(\mathrm{dT})_{\mathrm{n}},{ }^{22}$ cytosine in $\left.(\mathrm{dCdG})_{\mathrm{n}} \cdot(\mathrm{dCdG})_{\mathrm{n}}{ }^{24}\right)$ or from excimers formed by vertical stacking in $(\mathrm{dAdT})_{\mathrm{n}} \cdot(\mathrm{dAdT})_{\mathrm{n}}{ }^{21}$ However, their interpretation relying on the hypothesis that photons are absorbed by individual bases led to some peculiar conclusions. For example, the low values of fluorescence anisotropy determined for duplexes with respect to that observed for solutions of nucleotides was attributed to the fact that chromophores move faster within the double helices than when they are dissolved in bulk aqueous solution. ${ }^{25}$

The use of femtosecond spectroscopy, permitting to probe the early steps following UV absorption, where conformational changes of double helices are still restricted, can greatly contribute to elucidate many of the questions described above. In 2003, we reported the first time-resolved study of a DNA double helix with femtosecond resolution. ${ }^{26}$ The fluorescence of the oligomer $(\mathrm{dA})_{20} .(\mathrm{dT})_{20}$ was detected by means of the upconversion technique. It was observed that organization of nucleotides in single and double helices, $(\mathrm{dA})_{20},(\mathrm{dT})_{20}$ and $(\mathrm{dA})_{20} .(\mathrm{dT})_{20}$, renders the fluorescence decays progressively slower. However, the average decay time of $(\mathrm{dA})_{20} \cdot(\mathrm{dT})_{20}$ was still found to be very short $(1.23 \mathrm{ps}){ }^{26}$ As a next step, the investigation of the excited states and energy transfer in our laboratory, based on fluorescence detection, was focused on the much longer polymeric double helix poly(dA).poly(dT). ${ }^{1}$ This duplex appeared to be a much better model for fluorescence experiments than the oligomers because it can be obtained with the appropriate purity in a reproducible way. ${ }^{27}$ The time behaviour of the fluorescence detected for poly(dA).poly(dT), associated with the steady-state absorption and fluorescence spectra, was explained by the formation of FC states delocalized over several bases and subsequent energy transfer. ${ }^{1}$

In the mean time, Crespo-Hernández et al. studied the excited stated dynamics of oligomeric model helices using femtosecond transient absorption spectroscopy and detected important slow components (50-150 ps). ${ }^{28}$ They observed that this slow component of the transient signals recorded for $(\mathrm{dA})_{18} .(\mathrm{dT})_{18}$ is close to that determined for $(\mathrm{dA})_{18}$ but quite different from the one found for $(\mathrm{dAdT})_{9 .}(\mathrm{dAdT})_{9}$ and concluded that intrastrand excimers are 
formed in high yields. The discrepancy between excited state dynamics of such oligomers determined by transient absorption and fluorescence spectroscopy in the picosecond timescale triggered the discussion about the importance of dark states and the pertinence of separating base stacking and base pairing when addressing excited states dynamics in double helices. $^{29,30}$ The importance of interstrand stacking interactions has been underlined by a recent work based on refined calculations using the density functional theory augmented by London dispersion energies. $^{31}$

The model proposed by Crespo-Hernández et al. on the basis of transient absorption signals, detected at selected wavelengths, involves initial population of locally excited states which afterwards give rise to excimers. ${ }^{28,30}$ A quite recent transient absorption study by Fiebig and co-workers, performed also with femtosecond resolution, reported transient absorption spectra for similar duplexes and detected the fingerprint of exciton states. ${ }^{32}$ More precisely, they found that the relative intensity of a spectral feature around $435 \mathrm{~nm}$ increases with increasing the size of the oligomer. Such a behaviour being typical of a collective state, they concluded that the extent of the exciton in the examined duplexes exceeds four base-pairs.

Finally, it is important to stress that, besides controversial points arising from interpretation of experimental results obtained for DNA duplexes, others are simply due to experimental artefacts. Thus, in the discussion regarding the properties of the excited states of A-T duplexes, ${ }^{29,30}$ it has been mentioned ${ }^{30}$ that our findings concerning the fluorescence of oligomers do not agree with data published by other groups. ${ }^{33}$ In order to clarify these aspects, we pointed out specific difficulties encountered when handling DNA helices. ${ }^{27}$ These difficulties are related both to the quality of the DNA helices and their sensitivity towards UV radiation. We described in detail the experimental protocols which we developed in our laboratory to overcome these difficulties and we illustrated how the obtained data may be distorted by experimental pitfalls. We believe that a codification of rules, established by common agreement of the various groups working in the field, is vital so that comparison of the various experimental results can make sense.

\section{A case study: $\operatorname{poly}(d A) \cdot \operatorname{poly}(d T)$}

In this Section we describe results obtained by steady-state and time-resolved spectroscopy. It is important to take into account both sets of data before proposing a model describing the excited state relaxation processes in the examined double helix.

$\underline{\text { Steady-state spectra }}$ 
The absorption spectrum of poly $(\mathrm{dA})$.poly $(\mathrm{dT})$ presents three visible differences compared to the spectrum of the equimolar mixture of dAMP and TMP: a weak hypsochromic shift, an important hypochromism and a shoulder at $280 \mathrm{~nm}$ (Figure 1). The hypsochromic shift $\left(470 \pm 30 \mathrm{~cm}^{-1}\right)$ is explained by the fact that the dipolar coupling among $\pi-\pi *$ transitions of adenine and thymine gives rise to excited states extended over a few bases (Frenkel excitons). ${ }^{9}$ However, the important loss of oscillator strength $(50 \%)$ cannot be accounted for only by dipolar coupling. In contrast, interactions due to orbital overlap (charge transfer interactions between different chromophores) may give rise to hypochromism. ${ }^{34}$ Quantum chemistry calculations performed both by ZINDO $^{34}$ and TD-DFT ${ }^{35}$ methods for two stacked bases and Watson-Crick pairs predict the existence of interbase charge transfer transitions lying at energies lower than the $\pi-\pi^{*}$ transitions. Charge transfer states can be combined with Frenkel excitons and give rise to mixed excited states. ${ }^{35,36}$ Finally, the shoulder at $280 \mathrm{~nm}$ is interpreted as a fingerprint of charge transfer states and/or $n-\pi^{*}$ states. Such an attribution is inspired by a recent electroabsorption study which revealed the existence of strong Stark signals in the red wing of the spectra obtained for RNA single strands, and in particular in that of poly(A). ${ }^{37}$

Figure 1

The steady-state fluorescence spectra of poly(dA).poly(dT) do not change with the concentration when this is varied between $10^{-5}$ to $4 \times 10^{-3} \mathrm{M}$ per base. Moreover, the normalized spectra recorded for different excitation wavelengths between 245 and $285 \mathrm{~nm}$ do not present any noticeable difference (Figure 2). More precisely, any difference in the shape concerns less than $2 \%$ of the emitted photons. All the spectra in Figure 2 peak at $327 \mathrm{~nm}$, at somewhat shorter wavelength compared to the TMP $(330 \mathrm{~nm}) .{ }^{38}$ The similarity of the fluorescence spectrum of poly(dA).poly(dT) with that of TMP as well as the fact that the duplex spectrum does not change with the excitation wavelength was first reported by Ge and Georghiou. ${ }^{22}$ These authors reported also that the fluorescence quantum yield of this duplex increases significantly with the excitation wavelength, a fact that was not confirmed by our measurements. We found instead that $\phi$ is constant and equal to $(3.0 \pm 0.3) \times 10^{-4}$ for excitation wavelengths between 245 and $285 \mathrm{~nm}$. We stress that we used as a reference quinine sulfate dihydrate in $0.1 \mathrm{M} \mathrm{HCl}$ whose quantum yield has been shown to be constant $(\phi=0.59)$ independently of the excitation wavelength in the examined spectral region. ${ }^{39}$ 
The invariability in the shape of the steady-state emission spectra means that population of the excited states reached by excitation in the $245-285 \mathrm{~nm}$ range, leads always to emission from the same excited states. More importantly, the invariability of the fluorescence quantum yield for excitation in this spectral domain provides information about dark adenine excimers. If photons absorbed by adenine residues (assumed to act as "monomers") gave rise to dark (in the sense they cannot be detected in the steady-state fluorescence spectra) excimers, the fluorescence quantum yield should be lower at shorter wavelengths, where adenine has a relatively stronger absorbance (see inset in Figure 2). The variation in the fluorescence quantum yield being lower than our experimental error $(10 \%)$, we evaluate that such dark states are formed with a quantum yield lower than $10 \%$. This behaviour of the fluorescence spectra constitutes a first proof that energy transfer takes place among bases.

Figure 2

\section{Fluorescence decays}

The fluorescence decays of poly(dA).poly(dT) at 330, 380 and $420 \mathrm{~nm}$, covering a large temporal domain were presented in reference ${ }^{1}$. These were obtained by FU and TCSPC using the same excitation source (100 fs pulses at $267 \mathrm{~nm}$ ). In contrast to what is observed for each one of the monomeric chromophores, dAMP and TMP, whose fluorescence decays does not change with the fluorescence wavelength, the duplex signals show strong wavelength dependence.

Inspecting Figure 2 in ref. 1, it can be seen that the signals are deformed by the instrumental response functions, $c a .400 \mathrm{fs}$ (FU) and $50 \mathrm{ps}$ (TCSPC), respectively. In order to obtain a quantitative description of the decays and make comparison with literature data we performed a non-linear fitting/deconvolution procedure using multi-exponential functions: $\mathrm{F}(\mathrm{t})=\sum \alpha_{i} \exp \left(-t / \tau_{i}\right)$. For each wavelength, global fits were performed by merging the data obtained by FU and TCSPC. More precisely, we first fitted the FU signals by biexponential functions $\alpha_{1} \cdot \exp \left(-\mathrm{t} / \tau_{1}\right)+\alpha_{2} \cdot \exp \left(-\mathrm{t} / \tau_{2}\right)$ convoluted with the Gaussian apparatus function. Then, we defined an average time constant $\left\langle\tau_{1-2}>=\alpha_{1} \cdot \tau_{1}+\alpha_{2} \cdot \tau_{2}\right.$ which we used as a fixed parameter while fitting/deconvoluting the TCSPC data. The results of the fits are given in Table 1. Four exponentials are necessary in order to fit correctly the decays at $330 \mathrm{~nm}$ and $380 \mathrm{~nm}$ but at least one more time constant had to be added in order to obtain the same quality of fit for the decays at $420 \mathrm{~nm}$. Note that the shortest and the longest component are roughly 
similar for all three wavelengths, about 1 ps and $2.35 \mathrm{~ns}$, respectively. In contrast, the intermediate time constants, ranging from a few picoseconds to a few hundreds of picoseconds, show strong wavelength dependence.

Table 1

The relative amplitudes are all positive. This means that the rise time is shorter than $100 \mathrm{fs}$, which corresponds to the resolution of our setup after deconvolution. The shortest time constants, which are longer than those determined for dAMP and TMP (0.13 and 0.50 ps, respectively) ${ }^{38}$, have the largest amplitude.

The pre-exponential factors do not provide information about the distribution of the emitted photons as a function of time. Therefore, we have represented in Figure 3 the percentage of the total number of photons emitted during each decade of time. These values have been obtained from the deconvoluted decays. At $330 \mathrm{~nm}$ more than $80 \%$ of the photons are emitted in the first ten picoseconds but at $380 \mathrm{~nm}$ this value decreases to $45 \%$ and dwindles to a mere $18 \%$ at $420 \mathrm{~nm}$. At the latter wavelength, the long components are dominant and $64 \%$ of the photons are emitted at times longer than 100 ps. However, if the decays are scaled with respect to the relative intensity of the steady-state fluorescence spectrum at the corresponding wavelengths, we note that the important majority of the photons is emitted in less than $10 \mathrm{ps.}$

Figure 3

\section{Time-resolved fluorescence spectra}

Figure 4 shows the time-resolved fluorescence spectra obtained for poly(dA).poly(dT) on a wavenumber scale. They were recorded at $0.2 \mathrm{ps,} \mathrm{which} \mathrm{corresponds} \mathrm{to} \mathrm{the} \mathrm{maximum} \mathrm{of}$ the fluorescence intensity, and 1.2 ps by simultaneously scanning the monochromator wavelengths, rotating the upconversion crystal, and changing the delay line position in order to compensate for the group velocity dispersion. ${ }^{40}$

The time-resolved spectra are fitted with log-normal curves providing a quantitative description of the spectral shifts. At $0.2 \mathrm{ps}$, the spectrum peaks at $31300 \mathrm{~cm}^{-1}$ but one picosecond later, it has moved to lower energies by $1000 \pm 200 \mathrm{~cm}^{-1}$. At the same time interval the maximum spectrum recorded for TMP solutions is shifted less than $200 \mathrm{~cm}^{-1}$ showing that different relaxation process occur for the duplex. 
Figure 4

\section{$\underline{\text { Fluorescence anisotropy decays }}$}

Figure 5 shows the fluorescence anisotropy decays determined by $\mathrm{FU}$ for poly(dA).poly(dT) and an equimolar mixture of dAMP and TMP at $330 \mathrm{~mm}$. The low value of the fluorescence anisotropy determined for the polymer at zero time $(0.28 \pm 0.01)$ and its continuous decrease on the sub-picosecond time-scale constitute a second strong indication for energy transfer. When molecular motions are inhibited and the same electronic transition is involved in both absorption and emission, the anisotropy has a value close to 0.4. This is the case for TMP aqueous solutions. ${ }^{1}$ The slightly lower, albeit constant, value $(0.35)$ found for the dAMP and TMP equimolar mixture is due to the lower anisotropy of dAMP $(0.24)$ when it is excited at $267 \mathrm{~nm} .{ }^{38}$ It is worth-noticing that the fluorescence anisotropy of 2aminopurine incorporated in a double-stranded oligomer is constant on the sub-picosecond time-scale. ${ }^{41}$ This modified base, which forms a Watson-Crick pair with thymine, absorbs at lower energies than the natural bases. Consequently, when it is directly excited, no energy transfer can take place.

Figure 5

\section{A qualitative model for energy transfer}

Based on the steady-state spectra (Figures 1 and 2) and the fluorescence properties of poly $(\mathrm{dA}) \cdot \operatorname{poly}(\mathrm{dT})$ on the sub-picosecond time-scale, we propose a qualitative model describing energy transfer within this base sequence (Figure 6). Laser excitation at $267 \mathrm{~nm}$ populates a large number of excited states delocalized over a few bases. Internal conversion among these states (intraband scattering) takes place in less than 100 fs directing the population to the lower part of the exciton band. This part is located below $35000 \mathrm{~cm}^{-1}$ (wavelengths longer than $285 \mathrm{~nm}$ ) since excitation up to $285 \mathrm{~nm}$ at shorter wavelengths always leads to the same fluorescence spectrum. Intraband scattering is faster than the time resolution of our setup (100 fs after deconvolution) because we did not observed any rise time in the signals (Table 1). Here, we use the term "exciton band" to designate not only Frenkel excitons but also states having a partial charge transfer or $n \pi^{*}$ character which may be coupled through the phonon bath. 
Figure 6

The existence of FC delocalized excited states, postulated in our model, does not necessarily imply that all the emitting states probed by FU are also delocalized. It is possible that localization takes place during the intraband scattering. Indeed, the calculation of the FC excited states of the homopolymeric sequence, taking into account only dipolar coupling among $\pi-\pi *$ transitions, has shown that the excited states located at the bottom of the band are on average less delocalized compared to those at the middle of the band. ${ }^{6,8}$ The same type of calculations has also shown that during the intraband scattering the fluorescence anisotropy decreases (Figure 10 in ref. ${ }^{6}$ ) which accounts for the low initial value observed.

Assuming that emission stems from excited states localized on single thymine residues, as suggested by Ge and Georgiou, ${ }^{22}$ the wavelength dependence of the upconversion decays could be related to a different local solvation of each residue affecting the nonradiative decay processes. A comparison of our time-resolved spectra with the time-resolved spectra recorded for duplexes containing fluorescent probes, 2-aminopurine ${ }^{37}$ and coumarine $120,{ }^{42}$ pleads against this interpretation. The difference in the maxima observed at 0.2 and 1.2 ps for these probes, whose emitting states are localized, do not exceed $200 \mathrm{~cm}^{-1}$, whereas a value of $1000 \pm 200 \mathrm{~cm}^{-1}$ was found for poly $(\mathrm{dA})$. poly(dT). This observation is a strong indication that at least part of the emitting states is delocalized.

The precise identification of the various emitting states is intricate if we consider that delocalized states may be built with the participation of both dipolar coupling and interchromophore charge transfer interactions. When the charge transfer character of a low lying excited state increases its energy is expected to decrease and its lifetime to become longer. This is in agreement with the slowing down of the fluorescence decays upon increasing the emission wavelength (Figure 3). A further complication arises from the possible interference of $n-\pi^{*}$ transitions. The $n \pi^{*}$ state was experimentally shown to be the lowest in energy for adenine in the gas phase. ${ }^{43,44}$ When TMP and dAMP are dissolved in bulk water, the $n-\pi^{*}$ transitions shift to higher energies ${ }^{45,46}$ and ultrafast emission corresponding to the allowed $\pi-\pi^{*}$ transitions is observed. ${ }^{47-49}$ It would not be surprising that the specific environment created by the double helix, and in particular the presence of multiple electric charges on the phosphate moieties, affects the relative positions of the $n \pi^{*}$ and $\pi \pi^{*}$ states. Our fluorescence data obtained for poly(dA).poly(dT) does not allow us to distinguish between emission from $n \pi^{*}$ states or charge transfer states (excimer like). 
After the initial energy transfer via ultrafast intraband scattering, further energy transfer can also take place on longer time scales assisted by conformational motions of the double helix. Structural fluctuations have already been invoked in order to interpret efficient excitation transfer between adenine and 2-aminopurine in single stranded oligonucleotides. ${ }^{50}$ Such fluctuations occurring between close lying bases in double helices lead to modifications of the electronic coupling which is the driving force for energy transfer (Figures 2 and 4 in ref. $\left.{ }^{6}\right)$.

\section{Toward a quantitative description of energy transfer in double}

\section{helices}

The model illustrated in Figure 6 describes the energy transfer process in poly(dA).poly(dT) in a qualitative way. There are several problems to be resolved, from both an experimental and a theoretical point of view, before a more quantitative description of this process becomes possible.

A key point is to correlate the time-resolved spectroscopic signals with the evolution in time of the excited state population. The fluorescence decays shown in Figure 3 do not represent the survival probability of the excited states. According to the discussion in Section 4, photons are emitted from a variety of excited states: $\pi \pi^{*}$ states localized on single bases (adenine and/or thymine), Frenkel excitons delocalized over a few bases, charge transfer states, $\mathrm{n} \pi^{*}$ states, as well as combinations among them. Each one of these states is characterized by different radiative and non-radiative decay processes whose relative importance is not known. In the same way, the amplitude of transient absorption signals depends on the absorption cross-section of the probed excited states which is also expected to vary according to the type of the excited state.

The situation becomes even trickier due to the excitation transfer processes. In this respect, it is important to stress that, when energy transfer takes place within a low dimensional multichromophoric system, the time-constants derived from fits with multiexponential functions do not have physical meaning. ${ }^{51}$ The term "low dimensional system" designates a molecular system in which excitation energy is not transfer toward three dimensions with the same probability. This is clearly the case for a double helix where energy transfer can take place either along one strand or from one strand to the other with probabilities depending on the strength of the electronic coupling. The latter was shown to be site and conformation dependent. ${ }^{8,9}$ Therefore, the time constants reported in Table 1, as well 
as those determined by transient absorption, cannot be directly associated to the lifetime of a given excited state.

It is obvious that the understanding of energy transfer processes in DNA double helices will be greatly improved by obtaining data using various techniques. However, it is important to study the same double helix in the same conditions of buffer and ionic strength. As a matter of fact, base stacking is affected by salt concentration. ${ }^{52}$ Changes in structural parameters of the double helix will induce a variation of the electronic coupling which determines the properties of the excited states and, consequently, affect energy transfer. Moreover, the number of base-pairs in the duplex of a given base sequence is reflected in the excited state relaxation. Thus, the ratio of excitons compared to localized excited states, as determined from transient absorption spectra for $(\mathrm{dA})_{12}$. $(\mathrm{dT})_{12}$, differs from that found for

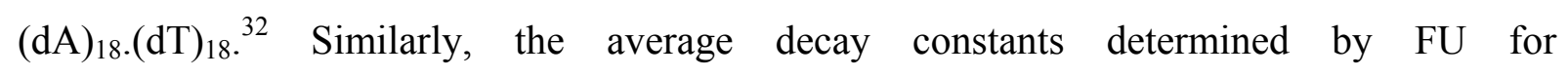
$\operatorname{poly}(\mathrm{dA}) \cdot \operatorname{poly}(\mathrm{dT})$ at $330 \mathrm{~nm}(2.1 \mathrm{ps}$; Table 1) is larger compared to that found for $(\mathrm{dA})_{20} .(\mathrm{dT})_{20}(1.2 \mathrm{ps}),{ }^{26}$ indicating also an increase in the degree of delocalization with the size of the duplex.

A first attempt to describe quantitatively excited state dynamics in poly(dA).poly(dT), including an interplay between Frenkel and charge transfer excitons, has been proposed recently by Bittner. ${ }^{53}$ However, experimental data cannot be directly compared to the reported numerical results, due to various approximations made in the calculations. In more general way, modeling of energy transfer in double helices has to take into account the plasticity of these systems associated to both the diversity of conformations and their dynamics during the lifetime of the excited states. This requirement is a serious obstacle for using high level quantum chemical calculations for the description of the excited states and their interactions. The development of approximations for the electronic coupling, whose validity will be tested with high level calculations for base-pairs and dimers of base-pairs, seems a promising direction to this end. 


\section{REFERENCES}

[1] D. Markovitsi, D. Onidas, T. Gustavsson, F. Talbot and E. Lazzarotto, Collective behavior of Franck-Condon excited states and energy transfer in double helices, J. Am. Chem. Soc., 2005, 127, 17130-31.

[2] I. Tinoco Jr., Hypochromism in polynucleotides, J. Am. Chem. Soc., 1960, 82, 478590.

[3] W. Rhodes, Hypochromism and other spectral properties of helical polynucleotides, $J$. Am. Chem. Soc., 1961, 83, 3609-17.

[4] T. Miyata and S. Yomosa, Exciton states in synthetic polynucleotides, J. Phys. Soc. Jpn., 1969, 27, 727-35.

[5] J. Eisinger and R.G. Shulman, Excited electronic states of DNA, Science, 1968, 161, 1311-19.

[6] B. Bouvier, J.P. Dognon, R. Lavery, D. Markovitsi, P. Millié, D. Onidas and K. Zakrzewska, Influence of conformational dynamics on the exciton states of DNA oligomers, J. Phys. Chem. B, 2003, 107, 13512-22.

[7] B. Bouvier, T. Gustavsson, D. Markovitsi and P. Millié, Dipolar coupling between electronic transitions of the DNA bases and its relevance to exciton states in double helices, Chem. Phys., 2002, 275, 75-92.

[8] E. Emanuele, D. Markovitsi, P. Millié and K. Zakrzewska, UV spectra and excitation delocalisation in DNA: influence of the spectral width, ChemPhysChem, 2005, 6, 1387-92.

[9] E. Emanuele, K. Zakrzewska, D. Markovitsi, R. Lavery and P. Millie, Exciton states of dynamic DNA double helices: alternating dCdG sequences, J. Phys. Chem. B, 2005, 109, 16109-18.

[10] M. Rist, H.-A. Wagenknecht and T. Fiebig, Exciton and excimer formation in DNA at room temperature, ChemPhysChem, 2002, 8, 704-07.

[11] D.F. Lewis, X. Liu, Y. Wu and X. Zuo, Stepwise evolution of the structure and the electronic properties of DNA, J. Am. Chem. Soc., 2003, 125, 12729-31.

[12] F.D. Lewis, L. Zhang, X. Liu, X. Zuo, D.M. Tiede, H. Long and G.C. Schatz, DNA as helical ruler: exciton-coupled circular dichroism in DNA conjugates, J. Am. Chem. Soc., 2005, 14445-53. 
[13] F.D. Lewis, Y. Wu, L. Zhang, X. Zuo and R.T.W. Hayes, R., DNA-mediated exciton coupling and electron transfer between donor and acceptor stilbenes separated by a variable number of base pairs, J. Am. Chem. Soc., 2004, 126, 8206-15.

[14] S.L. Shapiro, A.J. Campillo, V.H. Kollman and W.B. Goad, Exciton transfer in DNA, Opt. Comm., 1975, 15, 308-10.

[15] A. Anders, Energy transfer in nucleic acid-dye complexes, Opt. Comm., 1978, 26, 339-42.

[16] D.M. Rayner, A.G. Szabo, R.O. Loutfy and R.W. Yip, Singlet energy transfer between nucleic acid bases and dyes in intercalation complexes, J. Phys. Chem., 1980, 84, 28993.

[17] T.M. Nordlund, D. Xu and K.O. Evans, Excitation energy transfer in DNA: duplex melting and transfer from normal bases to 2- aminopurine, Biochem., 1993, 32, 1209095.

[18] D.-G. Xu and T.M. Nordlund, Sequence dependence of energy transfer in DNA oligonucleotides, Biophys. J., 2000, 78, 1042- 58.

[19] J. Cadet and P. Vigny, in H. Morrison (Ed.), Bioorganic Photochemistry. John Wiley \& Sons, New York, 1990, p. 1-272.

[20] P. Vigny and J.P. Ballini, in B. Pullman,N. Goldblum (Eds.), Excited states in organic chemistry and biochemistry. D. Reidel Publishing Company, Doordrecht, Holland, 1977.

[21] G. Ge and S. Georghiou, Excited-state properties of the alternating polynucleotide poly (dA-dT) poly (dA-dT), Photochem. Photobiol., 1991, 54, 301- 05.

[22] G. Ge and S. Georghiou, Room-temperature fluorescence properties of the polynucleotide polydA.polydT, Photochem. Photobiol., 1991, 54, 477- 80.

[23] S. Georghiou, G.R. Phillips and G. Ge, Resolution of the electronic absorption spectra of the adenine and thymine residues in poly(dA).poly( dT), Biopolymers, 1992, 32, 1417- 20 .

[24] C.-R. Huang and S. Georghiou, Room-temperature steady-state fluorescence properties of poly(dG-dC).poly(dG-dC), Photochem. Photobiol., 1992, 56, 95- 99.

[25] S. Georghiou, T.D. Bradrick, A. Philippetis and J. Beechem, Large-amplitude picosecond anisotropy decay of the intrinsic fluorescence of double-stranded DNA, Biophys. J., 1996, 70, 1909-22. 
[26] D. Markovitsi, A. Sharonov, D. Onidas and T. Gustavsson, Effect of molecular organisation in DNA oligomers studied by femtosecond fluorescence spectroscopy, ChemPhysChem, 2003, 3, 303-05.

[27] D. Markovitsi, D. Onidas, F. Talbot, S. Marguet, T. Gustavsson and E. Lazzarotto, UVB/UVC induced processes in model DNA helices studied by time-resolved spectroscopy: pitfalls and tricks, J. Photochem. Photobiol. A: Chem., 2006, 183, 1-8.

[28] C.E. Crespo-Hernández, B. Cohen and B. Kohler, Base stacking controls excited state dynamics in A-T DNA, Nature, 2005, 436, 1141-44.

[29] D. Markovitsi, F. Talbot, T. Gustavsson, D. Onidas, E. Lazzarotto and S. Marguet, Complexity of excited state dynamics in DNA, Nature, 2006, 441, E7.

[30] C.E. Crespo-Hernández, B. Cohen and B. Kohler, Nature, 2006, 441, E8.

[31] J. Řezáč and P. Hobza, On the nature of the duplex stability, Chem. Eur. J., in press.

[32] I. Buchvarov, Q. Wang, M. Raytchev, A. Trifonov and T. Fiebig, Electronic energy delocalization and dissipation in single- and double-stranded DNA, Proc. Natl. Acad. Sci., 2007, 104, 4794-97.

[33] R. Plessow, A. Brockhinke, W. Eimer and K. Kohse-Höinghaus, Intrinsic Time- and Wavelength-Resolved Fluorescence of Oligonucleotides: A Systematic Investigation Using a Novel Picosecond Laser Approach, J. Phys. Chem. B, 2000, 104, 3695-704.

[34] G.D. Scholes, Energy transfer and spectroscopic characterization of multichromophoric assemblies, J. Phys. Chem., 1996, 100, 18731-39.

[35] G.D. Scholes and K.P. Ghiggino, Electronic interactions and interchromophore excitation transfer, J. Phys. Chem., 1994, 98, 4580-90.

[36] T. Mančal, L. Valkunas and G.R. Fleming, Theory of exciton-charge transfer state coupled systems, Chem. Phys. Lett., 2006, 432, 301-05.

[37] S. Krawczyk and R. Lucowski, Electronic excited states of polynucleotides. A study by electroabsorption spectroscopy, J. Phys. Chem. B, 2007, 111, 1213-21.

[38] D. Onidas, D. Markovitsi, S. Marguet, A. Sharonov and T. Gustavsson, Fluorescence properties of DNA nucleosides and nucleotides: a refined steady-state and femtosecond investigation, J. Phys. Chem. B, 2002, 106, 11367- 74.

[39] R.A. Velapoldi and K.D. Mielenz: A fluorescence standard reference material: quinine sulfate dihydrate, U. S. Government Printing Office, Washington, D. C., Washington, 1980.

[40] T. Gustavsson, L. Cassara, V. Gulbinas, G. Gurzadyan, J.-C. Mialocq, S. Pommeret, M. Sorgius and P. van der Meulen, Femtosecond spectroscopic study of relaxation 
processes of three amino-substituted coumarin dyes in methanol and dimethyl sulfoxide, J. Phys. Chem. A, 1998, 102, 4229 -45.

[41] S.K. Pal, L. Zhao, T. Xia and A.H. Zewail, Site- and sequence-selective ultrafast hydration of DNA, Proc. Natl. Acad. Sci. USA, 2003, 100, 13746-51.

[42] D. Andreatta, J.L. Pérez Lustres, S.A. Kovalenko, N.P. Ernsting, C.J. Murphy, R.S. Coleman and M.A. Berg, Power-law solvation dynamics in DNA over six decades in time, J. Am. Chem. Soc. , 2005, 127, 7270-71.

[43] N.J. Kim, G. Jeong, J. Sung, Y.S. Kim and Y.D. Park, Resonant two-photon ionization and laser induced fluorescence spectroscopy of jet cooled adenine, J. Chem. Phys., 2000, 113, 10051-55.

[44] C. Canuel, M. Elhanine, M. Mons, F. Piuzzi, B. Tardivel and I. Dimicoli, Timeresolved photoelectron and photoion fragmentation spectroscopy study of 9methyladenine and its hydrates: a contribution to the understanding of the ultrafast radiationless decay of excited DNA bases, Phys. Chem. Chem. Phys., 2006, 8, 397887.

[45] T. Gustavsson, A. Banyasz, E. Lazzarotto, D. Markovitsi, G. Scalmani, M.J. Frisch, V. Barone and R. Improta, Singlet excited state behavior of uracil and thymine in aqueous solution: a combined experimental and computational study of 11 uracil derivatives, J. Am. Chem. Soc. , 2006, 128, 607-19.

[46] T. Gustavsson, N. Sarkar, E. Lazzarotto, D. Markovitsi, V. Barone and R. Improta, Solvent effect on the singlet excited state dynamics of 5-fluorouracil in acetonitrile as compared to water, J. Phys. Chem. B, 2006, 110, 12843-47.

[47] J. Peon and A.H. Zewail, DNA/RNA nucleotides and nucleosides: direct measurement of excited-state lifetimes by femtosecond fluorescence up-conversion, Chem. Phys. Lett., 2001, 348, 255-62.

[48] T. Gustavsson, A. Sharonov and D. Markovitsi, Thymine, thymidine and thymidine 5 '-monophosphate studied by femtosecond fluorescence upconversion spectroscopy, Chem. Phys. Lett., 2002, 351, 195-200.

[49] T. Gustavsson, A. Sharonov, D. Onidas and D. Markovitsi, Adenine, deoxyadenine and deoxyadenine 5'-monophosphate studied by femtosecond fluorescence upconversion spectroscopy, Chem. Phys. Lett., 2002, 356, 49- 54.

[50] J.M. Jean and B.P. Krueger, Structural fluctuations and excitation transfer between adenine and 2-aminopurine in single stranded deoxynucleotides, J. Phys. Chem. B, 2006, 110, 2899-909. 
[51] A. Blumen, J. Klafter and G. Zumofen, in I. Zschokke (Ed.), Optical spectroscopy of glasses. Reidel Publishing Co., 1986, p. 199-265.

[52] T. Douki, Low ionic strength reduces cytosine photoreactivity in UVC-irradiated isolated DNA, Photochem. \& Photobiol. Sci., 2006, 1045 -51.

[53] E.R. Bittner, Lattice theory of ultrafast excitonic and charge transfer dynamics in DNA, J. Chem. Phys., 2006, 125, 094909 (1-12).

[54] M. Riley, B. Maling and M.J. Chamberling, Physical and chemical characterization of two- and three-stranded adenine-thymine and adenine-uracil homopolymer complexes, J. Mol. Biol., 1966, 20, 359-89.

[55] P.O.P. Ts'o, A. Seymour, S.A. Rapaport and F.J. Bollum, A comparative study of polydeoxyribonucleotides and polyribonucleotides by optical rotatory dispersion, Biochem., 1966, 5, 4153-70. 
Table 1. Relative amplitudes and time constants (ps) derived from the fit of the fluorescence decays of poly $(\mathrm{dA})$.poly $(\mathrm{dT})$ by multi-exponential functions $\mathrm{F}(\mathrm{t})=\sum \alpha_{i} \exp \left(-t / \tau_{i}\right)$. A global fit is performed for the decays recorded at a given wavelength by fluorescence upconversion and time-correlated single photon counting.

\begin{tabular}{cccccccccccc}
\hline$\lambda(\mathrm{nm})$ & $\alpha_{1}$ & $\tau_{1}$ & $\alpha_{2}$ & $\tau_{\mathbf{2}}$ & $\left\langle\tau>_{\mathbf{1 - 2}}\right.$ & $\alpha_{\mathbf{3}}$ & $\tau_{\mathbf{3}}$ & $\boldsymbol{\alpha}_{\mathbf{4}}$ & $\tau_{\mathbf{4}}$ & $\boldsymbol{\alpha}_{\mathbf{5}}$ & $\tau_{\mathbf{5}}$ \\
\hline 330 & 0.59 & 0.85 & 0.41 & 3.9 & 2.10 & 0.00027 & 187 & - & - & 0.0001 & 2390 \\
380 & 0.75 & 1.14 & 0.25 & 6.5 & 2.48 & 0.00047 & 130 & - & - & 0.0006 & 2470 \\
420 & 0.70 & 0.94 & 0.27 & 8.3 & 2.99 & 0.0186 & 101 & 0.0015 & 480 & 0.0033 & 2200 \\
\hline
\end{tabular}




\section{Figure Captions}

Figure 1. Steady-state absorption spectra of poly(dA).poly(dT) (red), dAMP (short dashes), TMP (long dashes) and an equimolar mixture of dAMP and TMP (black). The molar extinction coefficient $(\varepsilon)$ of the duplex is given per base. ${ }^{54,55}$

Figure 2. Steady-state fluorescence spectra of poly(dA).poly(dT), normalized at $330 \mathrm{~nm}$ obtained following excitation at $245 \mathrm{~nm}$ (cyan), $255 \mathrm{~nm}$ (blue), $265 \mathrm{~nm}$ (dark yellow), 275 (green) and $285 \mathrm{~nm}$ (red). Concentration: $c a \cdot 10^{-3} \mathrm{M}$ per base. The sharp peaks are due to the Raman line of water. The inset corresponds to the difference of the absorption spectra of dAMP and TMP shown in Figure 1.

Figure 3. Percentage of the total emitted photons by poly(dA).poly(dT) at a given wavelength as a function of the time window.

Figure 4. Fluorescence spectra recorded by the upconversion technique for poly(dA).poly(dT) at $0.2 \mathrm{ps}$ (full squares) and 1.2 ps (open squares). Solid lines represent fits with log-normal functions. Excitation wavelength: $267 \mathrm{~nm}$.

Figure 5. Decay of fluorescence anisotropy obtained from the upconversion measurements at $330 \mathrm{~mm}$ for poly(dA).poly(dT) (red). For comparison the anisotropy of an equimolar mixture of dAMP and TMP (black) is also shown. Excitation wavelength: $267 \mathrm{~nm}$.

Figure 6. Schematic representation of the model proposed for the description of energy transfer in poly(dA).poly(dT) in the sub-picosecond time-scale. Excitation between 245 and $285 \mathrm{~nm}$ populates a large number of excited states delocalized over a few bases (area in grey). Internal conversion among these states (intraband scattering) taking place in less than $100 \mathrm{fs}$ brings the system to the lower part of the exciton band from which emission arises. The illustrated three band structures correspond to different duplex conformations. 


\section{Figure 1}

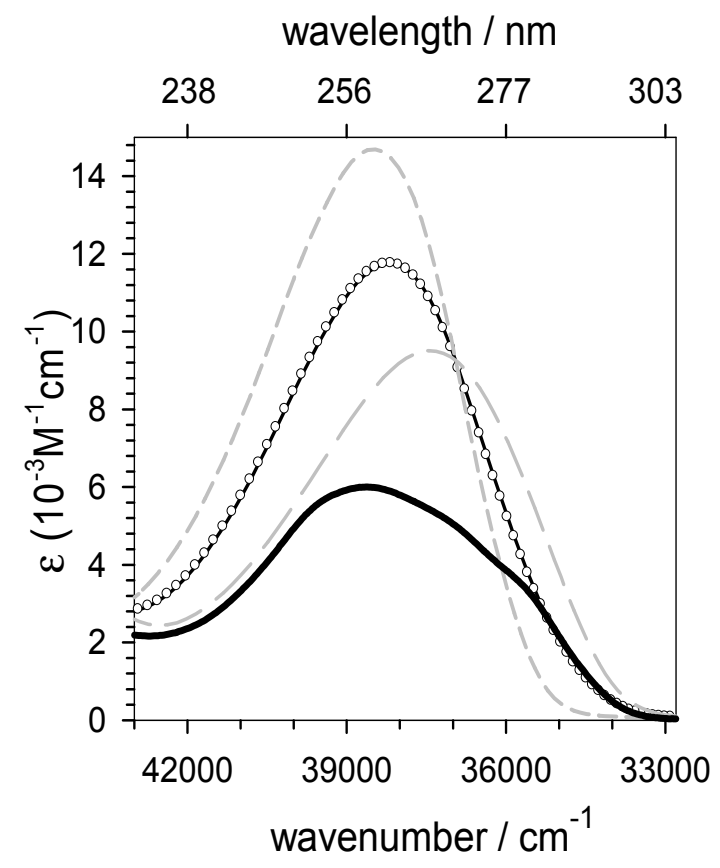

Figure 1. Steady-state absorption spectra of poly(dA).poly(dT) (solid line), dAMP (short dashes), TMP (long dashes) and an equimolar mixture of dAMP and TMP (circles). The molar extinction coefficient $(\varepsilon)$ of the duplex is given per base. ${ }^{54,55}$ 


\section{Figure 2}

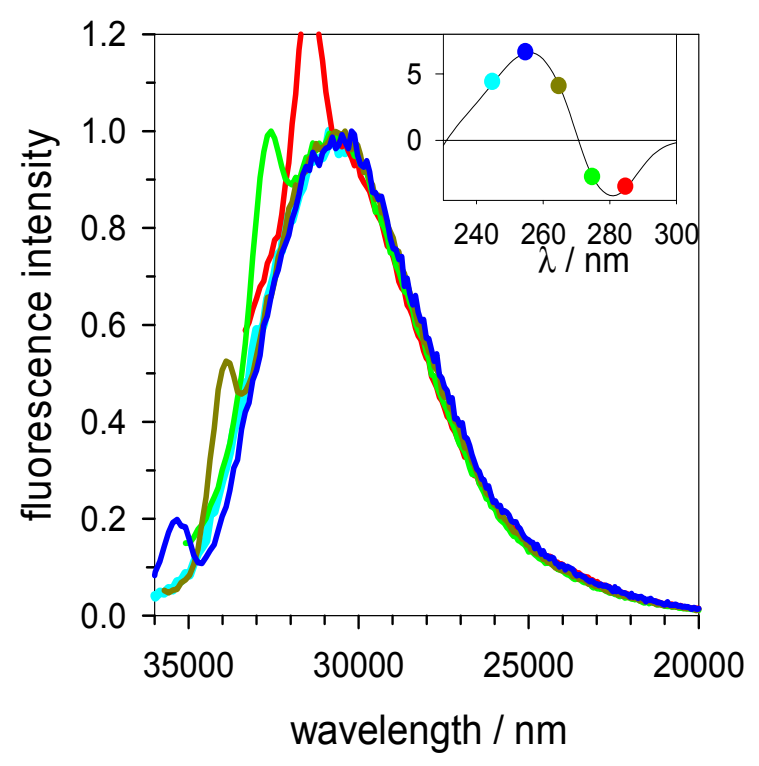

Figure 2. Steady-state fluorescence spectra of poly(dA).poly(dT), normalized at $330 \mathrm{~nm}$ obtained following excitation at $245 \mathrm{~nm}$ (cyan), $255 \mathrm{~nm}$ (blue), $265 \mathrm{~nm}$ (dark yellow), 275 (green) and $285 \mathrm{~nm}$ (red). Concentration: $c a \cdot 10^{-3} \mathrm{M}$ per base. The sharp peaks are due to the Raman line of water. The inset corresponds to the difference of the absorption spectra of dAMP and TMP shown in Figure 1. 
Figure 3

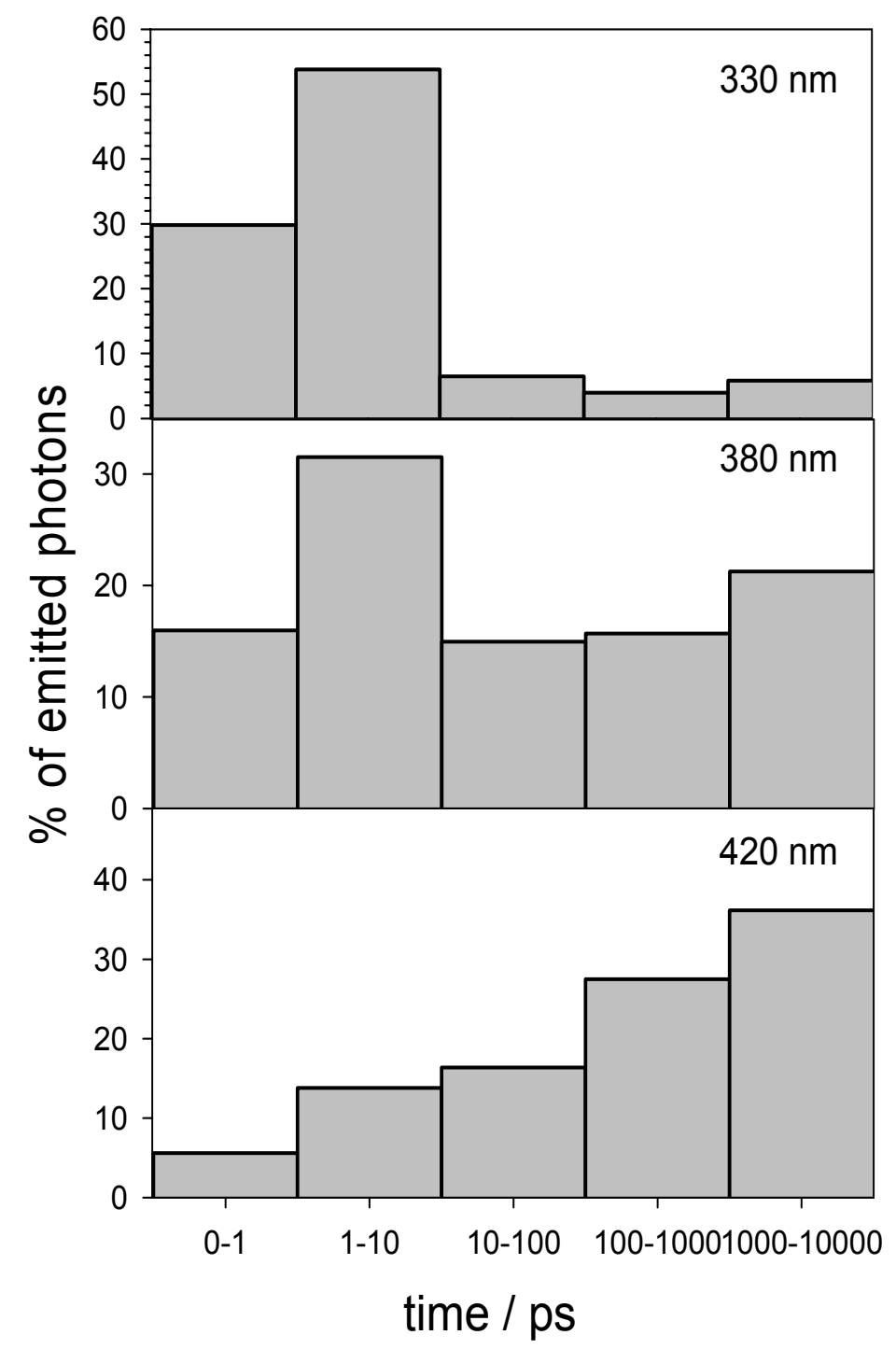

Figure 3. Percentage of the total emitted photons by poly(dA).poly $(\mathrm{dT})$ at a given wavelength as a function of the time window. 
Figure 4

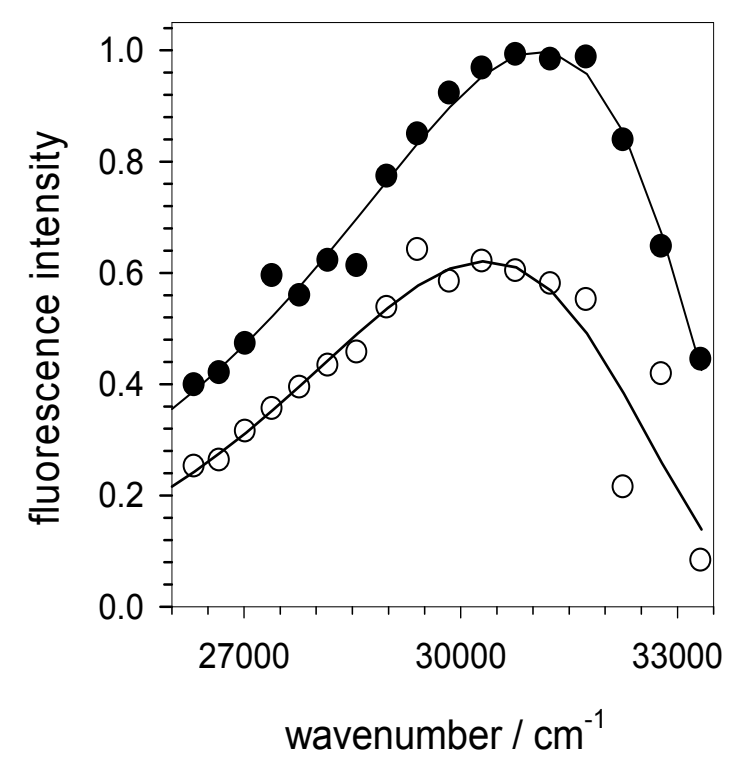

Figure 4. Fluorescence spectra recorded by the upconversion technique for poly(dA).poly(dT) at $0.2 \mathrm{ps}$ (full circles) and $1.2 \mathrm{ps}$ (open circles). Solid lines represent fits with log-normal functions. Excitation wavelength: $267 \mathrm{~nm}$. 


\section{Figure 5}

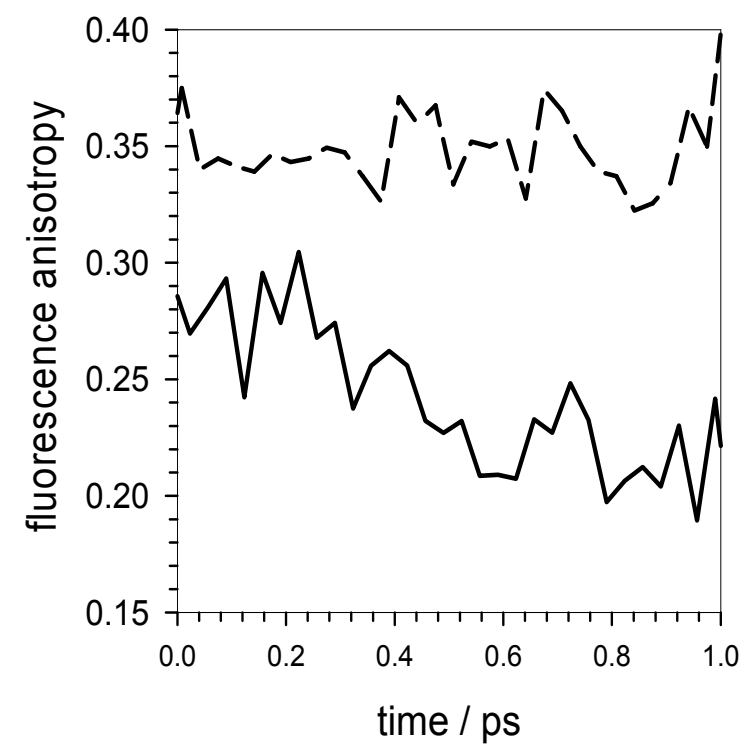

Figure 5. Decay of fluorescence anisotropy obtained from the upconversion measurements at $330 \mathrm{~mm}$ for poly(dA).poly(dT) (solid line). For comparison the anisotropy of an equimolar mixture of dAMP and TMP (dashed line) is also shown. Excitation wavelength: $267 \mathrm{~nm}$. 


\section{Figure 6}

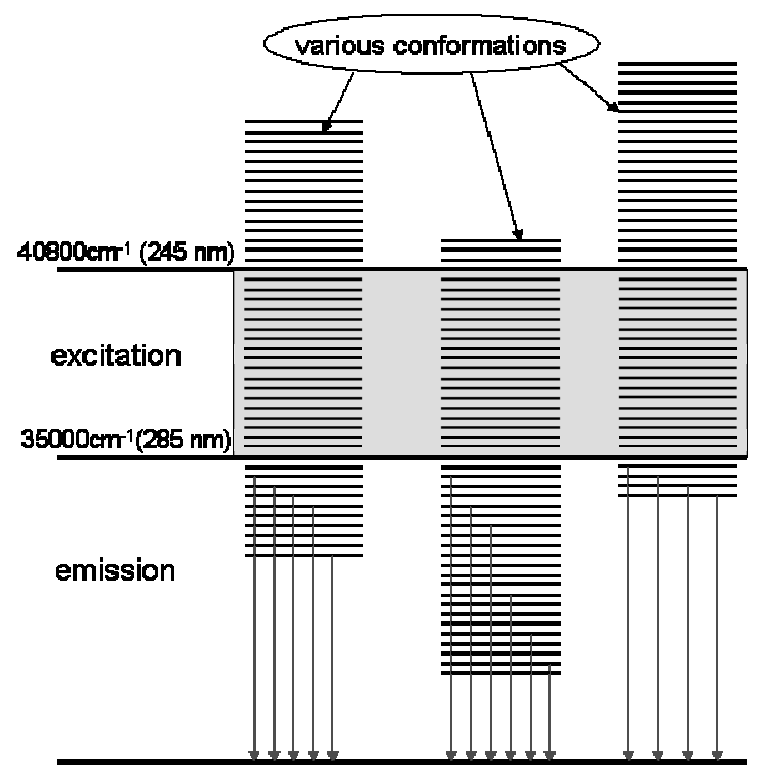

Figure 6. Schematic representation of the model proposed for the description of energy transfer in poly(dA).poly(dT) in the sub-picosecond time-scale. Excitation between 245 and $285 \mathrm{~nm}$ populates a large number of excited states delocalized over a few bases (area in grey). Internal conversion among these states (intraband scattering) taking place in less than $100 \mathrm{fs}$ brings the system to the lower part of the exciton band from which emission arises. The illustrated three band structures correspond to different duplex conformations. 


\section{TOC}

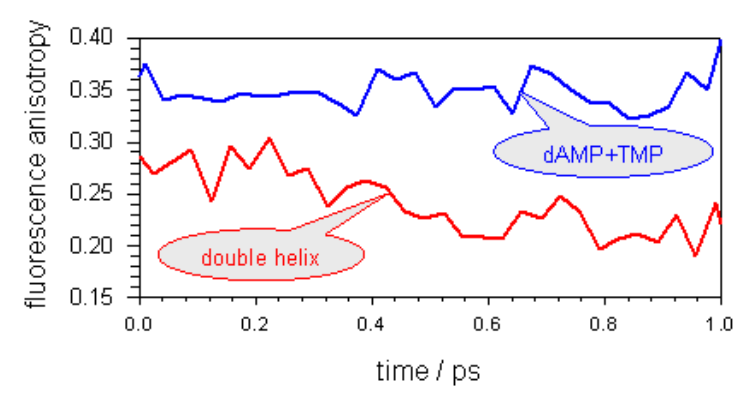

The article points out contentious questions regarding the nature of the excited states and the occurrence of energy transfer in DNA double helices and shows how they are currently approached. Using as example the polymer poly(dA).poly(dT), a model is proposed on the basis of time-resolved measurements (fluorescence decays, fluorescence anisotropy decays and fluorescence spectra, obtained with femtosecond resolution), associated to steadystate spectra. According to this qualitative model, excitation at $267 \mathrm{~nm}$ populates excited states that are delocalized over a few bases. Ultrafast internal conversion directs the excited state population to the lower part of the exciton band giving rise to fluorescence. Questions needing further investigations are underlined with particular emphasis on delicate points related to the complexity and the plasticity of these systems. 